Lexis Vol. XLII (1) 2018: 191-205

\title{
"Eso era mi meta de mí”: el doble posesivo en dos variedades de español amazónico*
}

\author{
Andrés Napurí \\ Pontificia Universidad Católica del Perú \\ Universidad del Pacífico
}

\section{RESUMEN}

Este trabajo revisa la presencia del doble posesivo en dos variedades de español amazónico del Perú (ISO 639-3: spq). Estas variedades son el resultado del contacto del español con dos lenguas amazónicas de familias y tipologías lingüísticas diferentes: la lengua bora de la familia lingüística bora y la lengua asháninka de la familia lingüística arawak. Por un lado, el doble posesivo está presente en la variedad del español amazónico con sustrato bora, pues esta lengua posee caso genitivo; en cambio, el doble posesivo no se presenta en la variedad de español amazónico con sustrato asháninka, ya que esta lengua no presenta el caso gramatical genitivo.

Palabras clave: bora, asháninka, español amazónico, doble posesivo, familia arawak, familia bora

\footnotetext{
"Este trabajo fue financiado por la Dirección de Gestión de la Investigación de la PUCP, a través de la subvención DGI-2017-1-0085. Una versión preliminar de esta nota fue presentada en el XV Congreso Internacional de la Asociación de Lingüistica y Filología de América Latina el 27 de julio de 2017.
}

https://doi.org/10.18800/lexis.201801.008 


\section{AbSTRACT}

This work reviews the presence of the double possession in two varieties of Peruvian Amazonian Spanish (ISO 639-3: spq). These varieties are the result of the contact of Spanish with two Amazonian languages of different families and typologies: Bora of the Bora linguistic family and Ashaninka of the Arawak linguistic family. On the one hand, the double possession is present in the variety of Amazonian Spanish with Bora substratum, since this language has a genitive case; on the other hand, the double possessive does not occur in the variety of Amazonian Spanish with Ashaninka substratum, as this language does not present the genitive grammatical case.

Keywords: Bora, Ashaninka, Amazonian Spanish, double possession, Arawakan languages, Boran languages

\section{Introducción}

El español amazónico del Perú (ISO 639-3: spq) reúne distintas variedades lingüísticas, las cuales son el resultado de un amplio contacto entre el español con lenguas amazónicas de familias y tipologías diferentes. Algunas características generales se han podido reconocer gracias al trabajo descriptivo de este grupo de variedades, pero todavía existen muchas interrogantes sobre particularidades diatópicas (Marticorena 2010; Ramírez 2003). En esa misma línea, no queda claro cuál es el grado de contacto que se puede establecer entre las distintas variedades de español amazónico y las lenguas amazónicas del Perú, ya que estos acercamientos se han visto eclipsados por descripciones más generales de estas variedades.

En ese sentido, conviene preguntarse en qué consisten estos rasgos generales y qué condiciones favorecen su aparición. Esta investigación revisará la presencia del doble posesivo en dos variedades del español amazónico. Por un lado, se explicitará algunas propiedades sintácticas del español amazónico de Pebas. En particular, se revisará el español amazónico que ha tenido contacto con la lengua bora de la familia bora (Seifart y Echeverri 2015; Aikhenvald 2012; Thiesen y Weber 2012). Estas lenguas presentan una fonología muy rica y bastante flexibilidad para la presentación del sujeto en los enunciados. 
Por otro lado, se describirá el español amazónico del Perené, para presentar una variedad que ha tenido contacto con la lengua asháninka de la familia lingüística arawak, cuya tipología es diferente.

En ambos casos se trabajarán con transcripciones y grabaciones de narraciones biográficas en busca de construcciones que presenten doble posesivo. Luego, se procederá a relacionar la presencia de esta partícula con la gramática de la lengua bora y la lengua asháninka respectivamente. Estas diferencias pueden ayudar a una precisión mayor de las variedades del español amazónico. Del mismo modo, nos puede dar una mejor imagen de en qué medida afectan las lenguas amazónicas al español regional.

\section{El multilingüismo en el territorio amazónico}

El multilingüismo en la Amazonía se diferencia del multilingüismo en el territorio andino. Esta diferencia se destaca en tanto las sociedades andinas reflejan un amplio espacio de contacto entre el español y el quechua o el aimara (Cerrón-Palomino 2000; Cerrón-Palomino 2008). Este bilingüismo data desde los primeros contactos con los españoles y se ha mantenido en la actualidad. Hoy en día, tiene mucha importancia el estudio del bilingüismo quechua-español en los programas de institucionalización del Ministerio de Educación (Mannheim 2015) y existe amplia literatura al respecto con enfoques más gramaticales o bien sociales (Zavala y Córdova 2010). La prominencia de las lenguas andinas en estos territorios también ha relegado el estudio del contacto con otras lenguas en los Andes (Emlen 2016). No obstante, existen trabajos puntuales que abordan el contacto del español andino con otras lenguas ya extintas, como el culle (Andrade 2016).

Por otra parte, para las personas en la Amazonía, el contexto es muy diferente (Emlen 2016). En gran medida, las sociedades amazónicas, hasta la fecha, han participado en redes multilingües entre dos o más lenguas indígenas. Estas redes se han visto favorecidas -no exclusivamente- por relaciones egalitarias entre distintos pueblos amazónicos. Un ejemplo interesante que podemos tomar 
en cuenta es el multilingüismo en el área del Vaupe en Colombia y en Río Negro en Brasil, donde miles de personas hablan varias lenguas dado que practican la exogamia lingüística (Aikhenvald 2012; Epps y Stenzel 2013; Jackson 1983). Si bien es frecuente que una persona hable varias lenguas, ${ }^{1}$ este individuo se identificará como parte de un solo pueblo indígena. Algunos estudiosos sugieren (Aikhenvald 1996; Epps 2006) que, a pesar de este multilingüismo, los hablantes evitan el cambio de código para mantener el estatus social de la lengua y el grupo humano. Consecuencia de ello, el léxico se mantiene en el tiempo, mientras existen procesos estructurales — semejanzas sintácticas - que se van convergiendo.

Del mismo modo, la presencia del español es tardía en la Amazonía. El contacto más intenso entre las lenguas amazónicas y el español se da recién a inicios del siglo XX con el boom del caucho promovido por la Corona inglesa y los Estados republicanos. Es innegable la presencia dominante del español en estos territorios sea para acceder a los servicios del Estado o bien para tener éxito en la escuela; sin embargo, muchas lenguas amazónicas mantienen espacios exclusivos y se siguen transmitiendo a las generaciones más jóvenes. ${ }^{2}$ Sin duda, la presencia de las lenguas amazónicas en el futuro dependerá de en qué medida son útiles para los hablantes para acceder a un mejor estatus social o el valor cultural que tenga aprender una lengua europea.

\section{Sobre el español amazónico y las lenguas de sustrato}

Las relaciones de parentesco que se establecen en la Amazonía peruana son fundamentales para abordar las características del español amazónico (ISO 639-3: spq). En este breve trabajo se destacarán dos variedades para lo cual es importante tener una idea de cuál es el sustrato de las lenguas indígenas que se encuentran presentes. Por un lado, los ejemplos del español amazónico de Loreto

Lenguas tariana, tukano y nheengatú

2 Para el caso del bora, se habla entre niños de siete y ocho años; el shawi también se transmite entre pequeños. 
corresponden a la variedad de un hablante de la lengua bora; por otro lado, la variedad del español amazónico del Perené es la de un hablante de lengua asháninka.

La lengua bora (ISO 639-3: boa) forma parte de la familia lingüística bora (Seifart y Echeverri 2015; Aikhenvald 2012) y se extiende entre la Amazonía peruana y colombiana. Es una lengua aglutinante que muestra mucha libertad en el orden de sus constituyentes: bien puede ser SOV o OSV. El sujeto puede ser nulo y presenta género en el sistema pronominal; sin embargo, no requiere concordancia en la construcción de frases nominales. La lengua asháninka (ISO 639-3: cni) forma parte de la familia lingüística arawak (Aikhenvald 2012) y abarca tanto la Amazonía peruana como brasilera. Es una lengua aglutinante y polisintética. Posee cierta flexibilidad por el orden de palabras, con cierta preferencia por VSO. Presenta sistema de género en el que predomina no-masculino y se suele expresar en construcciones con 3sG. Los marcadores de género son más productivos en asháninka, en tanto se presenta en nominalizaciones por yuxtaposición y sufijos (Mihas 2010). Una diferencia que considero importante para esta investigación es que el bora - a diferencia del asháninka - tiene marca de caso genitivo. En asháninka, existen construcciones posesivas que bien pueden interpretarse como construcciones genitivas o de pertenencia, pero no presenta un caso gramatical como sí sucede con la lengua de la Amazonía noreste peruana.

\subsection{Genitivo en la lengua bora}

De acuerdo con Thiesen y Weber, el genitivo unifica al poseedor con el poseído en una sola palabra fonológica. Es decir, los procesos fonológicos que afectan a las frases nominales afectan a ambos lexemas como una sola unidad. Así mismo, entre el poseedor y el poseído se asigna un tono bajo (2012: 251-267).

$$
\left.{ }_{\mathrm{SN}} \mathrm{SN}_{\text {poseedor }} \mathrm{N}_{\text {poseído }}\right]
$$

Estos procesos fonológicos van desde la asignación de tono como a la armonización vocálica. A continuación, presentaré algunos 
ejemplos sencillos para identificar cómo se expresa el genitivo en la lengua bora. Lo importante en estos ejemplos es que podemos reconocer que la gramática de la lengua opera tras la combinación de los ítems léxicos.

a.

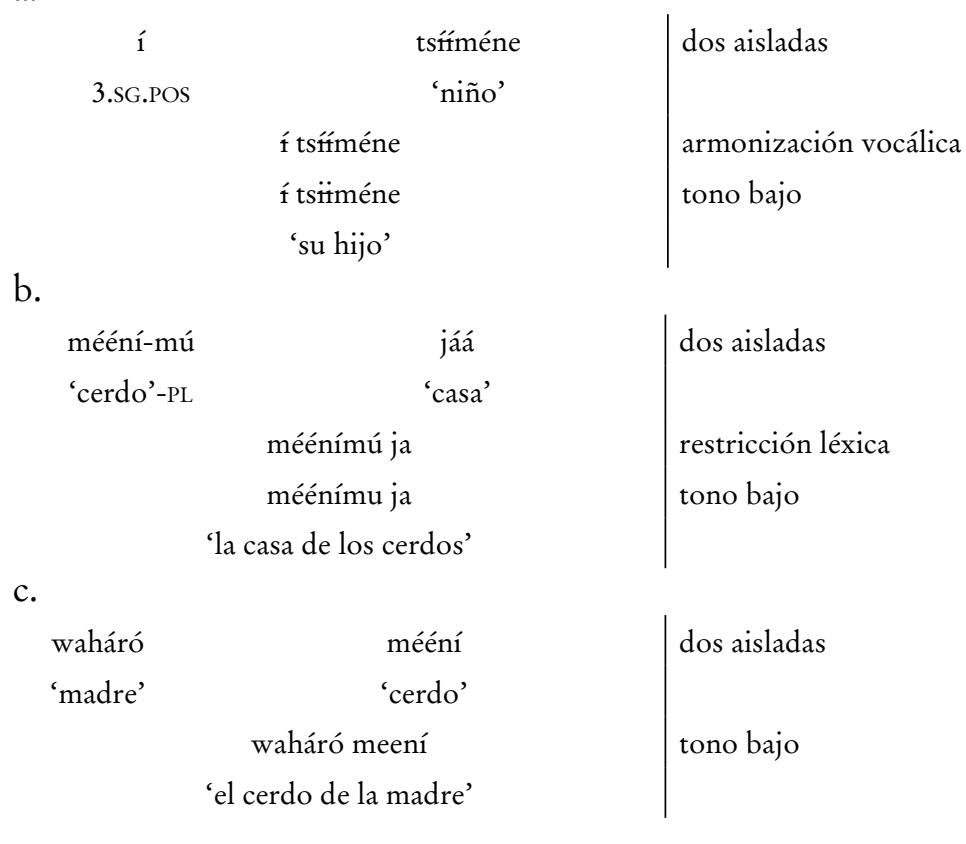

b.

Es importante notar que la operación de este tono bajo aparece además de las posibles construcciones con pronombres reflexivos o posesivos que posee la lengua bora. Es posible que un hablante bora emplee construcciones posesivas sin sentido genitivo. Estos ejemplos ya exceden el propósito de esta investigación, pero se pueden consultar en Thiesen y Weber (2012). En ese sentido, la lengua bora emplea el tono para codificar gramaticalmente el caso genitivo.

El efecto del caso genitivo de la lengua bora en el español amazónico ya ha sido estudiado por otros autores (RodríguezMondoñedo y Fafulas 2016). En el análisis que ellos recogen existía una fuerte presencia de construcciones con doble posesivo. 
d. "Para qué, quezás, quería aprender más dialecto viviendo con su papa de mis dos hijos porque yo ya estaba (e)n practicando, y ya no he podido más, pues, cuando me salió de su lado de ellos.” (Rodríguez-Mondoñedo y Fafulas 2016: 343).

De acuerdo con ellos, la presencia del doble posesivo se debe principalmente a dos razones: la presencia del caso genitivo en la gramática del bora y la permanencia de una variedad antigua de español (Rodríguez-Mondoñedo y Fafulas 2016: 349). Esta afirmación es relevante para la discusión que se plantea continuar líneas más adelante.

\subsection{El asháninka no presenta caso genitivo}

Para los comentarios relacionados a la gramática de la lengua asháninka me referiré al trabajo de Elena Mihas, quien sustentó en el año 2010 su tesis doctoral Essentials of Ashéninka Perené Grammar. Esto es de particular importancia porque la persona involucrada en esta investigación nació y vivió su juventud en las riberas del río Tambo, próximo al río Perené. La denominación de ashánika y ashéninka responde sobre todo a divisiones políticas más que lingüísticas: ambas denominaciones bien son variedades de una misma lengua.

En la variedad del asháninka del Perené no existe caso genitivo. Si bien es posible realizar construcciones con sentido genitivo, estas son típicamente frases nominales sin que signifique un proceso derivacional en el resultado, como sí sucede en la lengua bora. Los siguientes ejemplos (Mihas 2010) revelan que existe un sistema pronominal para marcar la posesión de un elemento.

e.

$\begin{array}{cccc}\text { noime } & \text { avani } & \text { ipyaameni } & \text { oishi } \\ \text { no=ime } & \mathrm{a}=\text { vani } & \mathrm{i}=\text { pyaameni } & \mathrm{o}=\mathrm{ishi} \\ \text { 1.SG.POSS='esposo' } & \text { 1.PL.POss='jardín' } & \text { 3.M.SG.POss='arco' } & \text { 3.F.SG.POSS='pelo' } \\ \text { 'mi esposo' } & \text { 'nuestro jardín' } & \text { 'su }_{(\mathrm{m})} \mathrm{arco}^{\prime} & \text { 'su }_{(\mathrm{F})} \text { pelo' }\end{array}$


Es importante notar, además, que este sistema pronominal posesivo es sensible al género. Existen algunos procesos fonológicos que operan en ejemplos de Mihas (2010); sin embargo, la autora señala que no son exclusivos de las construcciones posesivas. En este punto, conviene explicitar que interesa saber en qué medida este sistema de posesión puede afectar al español amazónico que hablan las personas con sustrato asháninka.

Entre los ejemplos que se presentan en (e) hay una precisión que es importante destacar. Entre las lenguas amerindias existen estrategias particulares para marcar elementos inalienables (Aikhenvald 2012), los cuales suelen ser típicamente las partes del cuerpo. A menos que sea un caso de desmembramiento, la 'mano', 'cabeza' o 'pelo' siempre le pertenecen a una persona; por consiguiente, lo regular es expresar estas palabras siempre con alguna marca de posesión. De acuerdo con Mihas (2010), las estrategias para referirse a un miembro del cuerpo en la lengua asháninka —en este caso pelo- no se diferencia de otras construcciones posesivas.

\section{Sobre las variedades analizadas para esta investigación}

Esta investigación se respalda en la revisión de dos materiales. El primero es la historia de vida de Hilario Díaz Peña (2017), maestro bora que nació en la comunidad de Brillo Nuevo, distrito de Pebas, provincia Mariscal Castilla, región Loreto. Esta historia de vida recoge más de doce horas de grabaciones. Las transcripciones —durante su edición - respetaron su variedad de español amazónico, así como los distintos cambios de código que realizó.

Conviene señalar que Díaz Peña es un individuo multilingüe: domina bora, español, asháninka, shipibo y otras lenguas. También es un ejemplar de la exogamia amazónica, pues su esposa es del pueblo indígena shipibo. Si bien Díaz Peña domina varias lenguas, durante su infancia y adultez temprana solo habló dos: bora y español. Luego, tras su formación como docente, tuvo que aprender el shipibo y el asháninka para trabajar en distintas comunidades amazónicas. 
El segundo material corresponde a una serie de transcripciones que corresponden a cerca de doce horas de entrevistas con Raúl Casanto (2017), líder histórico del pueblo asháninka. La biografía lingüística de Casanto es distinta a la de Díaz Peña. Él nació en Puerto Bermúdez, provincia de Oxapampa, región Pasco. Sin embargo, desde muy joven vivió en las riberas del río Perené hasta cumplir treinta y nueve años. Luego, por trabajo, se mudó a Ucayali por unos años. Actualmente vive en una comunidad asháninka desde hace ya unos diecisiete años.

La metodología empleada para la grabación de estos materiales es muy similar a la empleada con el caso anterior: es el mismo equipo de investigadores. Idealmente, las preguntas formuladas durante estas investigaciones servían como marco para que el entrevistado se explaye acerca de sus vivencias y momentos importantes sobre su vida. No se han realizado preguntas que cotejen concretamente el uso de doble posesivo, pero durante las horas de grabaciones se han ido expresando de manera natural de acuerdo a la narración.

\section{Análisis de los materiales}

\subsection{Español amazónico con sustrato bora}

Como bien se puede prever por la investigación de RodríguezMondoñedo y Fafulas (2016), el español de Díaz Peña (2017) posee varias expresiones con doble posesivo. Estas aparecen de manera regular a lo largo de toda su narración. Se presentan de manera sistemática cuando Díaz Peña expresa algún tipo de pertenencia, sea afectiva o material.

f. "Entonces, ya quizás mi mamá tenía planes con el amante, que es mi padrastro ahora (lo estimo bastante; ya pues, qué se va a hacer, su querer de mi mamá)gg” (Díaz Peña 2017: 71)

g. "«Yo quiero otra cosa; no quiero estar ahí. Quiero más allá». Eso era mi meta de mí, propio.” (Díaz Peña 2017: 91)

h. "Entonces, yo de decir que mi papá ha nacido allá, yo estaría mintiendo pues. No nacimos en Colombia nosotros. Sí nació 
mi tía y mi abuelo, su papá de mi tía, su papá de mi mamá.” (Díaz Peña 2017: 121)

i. "A veces en la hamaca. Por eso es que yo me retiro de su casa de mi mamá, porque no había ningún apoyo para mí. (Díaz Peña 2017: 111)

j. "Le cavas bonito y de ahí tomas su agua de su poco semillitas que tiene.” (Díaz Peña 2017: 197)

k. "Hicimos nuestros cushmas ${ }^{3}$ de su cogollo del aguaje." (Díaz Peña 2017: 253)

Es importante notar —en esta revisión-que es posible sostener que la presencia del genitivo en la lengua bora haya favorecido la necesidad de identificar un "receptor" para que sea expresado en español (Rodríguez-Mondoñedo y Fafulas 2016). De esta manera, influye sobre el determinante "el" o "la" para que se manifieste como “su”. ${ }^{4}$ Es posible, además, que el contacto con otras variedades de español favorezca la aparición de este doble posesivo. En el caso de Díaz Peña (2017), él vive una buena parte de su infancia cerca de Iquitos y es posible que haya estado en contacto con otras variedades de español que tengan también este rasgo. Esta posibilidad se ha visto contemplada en el español andino y bien podría darse el caso en el español amazónico (De Granda 1997). Sin embargo, en la hipótesis del español andino, también se reconoció que la gramática de la lengua andina era relevante para la aparición del doble posesivo; es decir, era un ejemplo análogo.

\subsection{Español amazónico con sustrato asháninka}

Tras la revisión de las transcripciones del español amazónico con sustrato asháninka, no se encontró más que un caso de doble posesivo —ejemplo (m)—; más bien, las narraciones de Raúl Casanto

\footnotetext{
Túnica tradicional de los pueblos amazónicos, tradicionalmente de algodón.

A manera de comentario, conviene señalar que de manera intuitiva se reconoce que el español amazónico de hablantes con sustrato shipibo también expresa doble posesivo. El shipibo también tiene caso genitivo.
} 
(2017) expresan la posesión de manera similar a otras variedades de español, como el costeño. A continuación, algunos ejemplos:

1. "También llegaba a la casa de mi abuelo y, bueno pues, eran funcionarios del gobierno y otros que no eran."

m. "porque la lucha de mi abuelo era justo por la reivindicación de las tierras, por tener más educación, más escuelas en las comunidades. Esa era su lucha de mi abuelo.”

n. "Yo he crecido prácticamente al lado de mi abuelo, en sus andanzas; mi abuelo era más político que religioso."

o. "Se ha hecho pasar por personal de mi abuelo y se llevó todas las cositas que teníamos en el hotel, nuestra ropa, los documentos, todo."

p. "Queremos que los pueblos desarrollen y sobre todo en el tema económico, que cada uno sea independiente de su economía, independiente de su religión y de su cultura y de todo lo demás"

q. "Por ejemplo, algunos paisanos decían, cuando le entregan el título de propiedad de sus territorios"

Llama mucho la atención que el único caso en que se presente el doble posesivo sea tras una aclaración. Uno puede interpretar que es un caso de énfasis o bien una expresión posible dentro de la gramática, mas no favorecida por esta misma. En este caso también conviene señalar que Casanto no ha tenido durante muchos años contacto con otras variedades de español. Del mismo modo, tampoco ha vivido en espacios con mucha circulación o comercio: la mayor parte de su vida ha estado entre comunidades asháninkas.

\section{Otros rasgos compartidos y cierre}

Entre ambas variedades es posible identificar rasgos gramaticales comunes. Por ejemplo, tanto Díaz Peña (2017) como Casanto (2017) expresan variedades leístas de español. Esto no significa que estos hablantes no identifiquen el género en los clíticos, pero no lo expresan de igual manera que en otras variedades. 
Estas diferencias, en parte, se pueden explicar porque las lenguas bora y asháninka codifican el género de manera diferente que el español. De esta manera, la interpretación del género en los clíticos resulta una tarea muy difícil. En esa misma línea, ambas variedades de español amazónico expresan varios casos de discordancia de género. Tanto la lengua bora como la lengua asháninka no requieren de ningún tipo de concordancia a nivel de sintagma nominal. Esta exigencia es nueva y exclusiva del español. Por consiguiente, es plausible que ambas variedades de español amazónico tengan dificultades con la concordancia entre modificadores $y$ núcleos de sintagma nominal.

Estos rasgos forman parte del estereotipo que se ha construido de forma peyorativa contra el español amazónico (Jara Yupanqui 2013; Jara Yupanqui y Valenzuela 2013). Como todo prejuicio, es una representación limitada y poco fiel de la realidad del español amazónico. Al menos en este breve repaso, se ha podido identificar que un rasgo que parecía ser común y convergente en el español de la Amazonía peruana - el doble posesivo- resulta estar estrechamente vinculado con la gramática de la lengua de sustrato que está en contacto con el español: si la lengua tiene una estructura gramatical que favorezca la marca de pertenencia, es plausible que el español amazónico exprese doble posesivo, como es para el caso de lenguas que presentan caso genitivo.

\section{Referencias bibliográficas}

Aikhenvald, Alexandra Y.

1996 "Areal Diffusion in North-West Amazonia: The Case of Tariana”. Anthropological Linguistics. 38, 73-116.

2012 The Languages of the Amazon. Oxford: Oxford University Press. https://doi.org/10.1093/acprof

ANDRADE, Luis :oso/9780199593569.001.0001

2016 The Spanish of the Northern Peruvian Andes: A Sociohistorical and Dialectological Account. New York: Peter Lang Verlag. https://doi.org/10.3726/978-3-0353-0800-6 
CASANTO, Raúl

2017 “Entrevistas para la elaboración de su historia de vida”. [En transcripción y proceso de edición]. Lima, 15 de junio.

Cerrón-Palomino, Rodolfo

2000 Lingüística aimara. Cuzco: Centro de Estudios Regionales Andinos Bartolomé de las Casas.

2008 Quechumara: estructuras paralelas del quechua y del aimara. Segunda edición. La Paz: Plural.

De Granda, Germán

1997 "Replanteamiento de un tema controvertido: Génesis y retención del doble posesivo en el español andino". Revista de Filología Española. 77, 139-147. https://doi.org/10.3989/ rfe.1997.v77.i1/2.368

Díaz Peña, Hilario

2017 No estoy viajando callado: historia de vida de un maestro boral Tsá cúvébulléré o pébijkyatúne: bóórámú uwáábóóbe ijkyá uubálle. Eds., L. Andrade, E. Delgado, S. Frisancho, y A. Napurí. Trans., W. Panduro. Lima: Fondo Editorial de la PUCP.

EMLen, Nicholas Q.

2016 "Multilingualism in the Andes and Amazonia: A View from In-between: Multilingualism in the Andes and Amazonia". The Journal of Latin American and Caribbean Antbropology. https://doi.org/10.1111/jlca.12250

Epps, Patience

2006 “The Vaupe's Melting Pot: Tucanoan Influence on Hup”. En Grammars in Contact: A Cross- Linguistic Typology. A. Y. Aikhenvald y R. M. W. Dixon. Oxford: Oxford University Press, 267-289.

Epps, Patience y Stenzel, Kristine

2013 "Introduction”. En Upper Rio Negro: Cultural and Linguistic Interaction in Nortbwestern Amazonia. Eds., P. Epps y K. Stenzel. Río de Janeiro: Museu Nacional do Indio FUNAI, 13-50. 
JACKSON, Jean Elizabeth

1983 The Fish People: Linguistic Exogamy and Tukanoan Identity in Northwest Amazonia. Cambridge: Cambridge University Press. https://doi.org/10.1017/CBO9780511621901

JARA YUPANQUi, Margarita

2013 "Peruvian Amazonian Spanish: linguistic variation, social meaning and identities”. Sociolinguistic Studies. 6, 3. https:// doi.org/10.1558/sols.v6i3.445

Jara Yupanqui, Margarita y Valenzuela, Pilar M.

2013 "El uso del perfecto en secuencias narrativas en el español peruano amazónico: el caso de Jeberos”. Lexis. XXXVII, 1, $33-70$.

ManNheim, Bruce

2015 “All Translation Is Radical Translation”. En Worlds: The Epistemological Space of Translation. Trad., C. Severi y W. F. Hanks. Chicago: HAU Books, 199-219.

Marticorena, Manuel

2010 El castellano amazónico del Perú. Iquitos: Instituto de Investigaciones Educativas e Históricas de la Amazonía Peruana.

Minas, Elena

2010 Essentials of Ashéninka Perené Grammar. Tesis Doctor of Philosophy in Linguistics. Milwaukee: University of Wisconsin.

RAMíreZ, Luis Hernán

2003 El español amazónico hablado en el Perú: hacia una sistematización de ese dialecto. Lima: Juan Gutemberg

Rodríguez-Mondoñedo, Miguel y Fafulas, Stephen

2016 "Double possession in Peruvian Amazonian Spanish". En Inquiries in Hispanic Linguistics. From theory to empirical evidence. Eds., A. Cuza, L. Czerwionka, y D. J. Olson. New York: John Benjamins Publishing Company, 335 -354 https:// doi.org/10.1075/ihll.12.18rod

Seifart, Frank y Echeverri, Juan Alvaro

2015 "Proto Bora-Muinane”. Línguas Indígenas Americanas. 15, 2, 279-311. 
Napurí • El doble posesivo en dos variedades de español amazónico 205

Thiesen, Wesley y Weber, David

2012 A Grammar of Bora with Special Attention to Tone. Dallas: SIL International.

Zavala, Virginia y Córdova, Gavina

2010 Decir y callar. Lenguaje, equidad y poder en la universidad peruana. Lima: Fondo Editorial de la PUCP.

Recibido: 26/12/2017

Aceptado: 27/04/2018 